\title{
Low-Order Approximations for the Normal Probability Integral and the Error Function
}

\author{
by David G. Carta
}

\begin{abstract}
Rational fractions of the form $0.5 /(a+b x+\ldots)^{2 q}$ are used to evaluate the function of interest. Polynomials of from third to sixth order are derived which achieve absolute errors ranging from 0.01 to 0.000001 for all (real) positive $x$, and relative errors of from 0.1 to 0.00001 for (real) positive $x$ less than 3.1, 4.0, and 5.2. Denominator coefficients are calculated by linearizing the rational fraction about progressively improved nominal solutions and using linear programming to solve the resulting linear minimax problems.
\end{abstract}

1. Introduction. In the course of deriving rapidly executable approximations for a real-time processing application [1], it became necessary to find a low-order method for evaluating the normal probability integral*

$$
Q(x)=\frac{1}{\sqrt{2 \pi}} \int_{x}^{\infty} \exp \left(-t^{2} / 2\right) d t
$$

A review of the literature yielded only one approximation suitable for low-order application. This was of the following form from Hastings [2]:

$$
Q(x)=0.5 /\left(1+c_{1} x+c_{2} x^{2}+c_{3} x^{3}+c_{4} x^{4}\right)^{4}+e(x),
$$

$$
|e(x)| \leqslant 0.00025, \quad 0 \leqslant x<\infty .
$$

While this approximation was not entirely suited to our needs, the reciprocal polynomial form of the approximation seemed eminently well chosen. All approximations developed in this paper will be of a similar form:

$$
\bar{Q}(x, c)=0.5 /\left(c_{1}+c_{2} x+\ldots+c_{n} x^{n-1}\right)^{2^{q}} .
$$

Note the added flexibility of a variable first coefficient, as opposed to the fixed value of unity by Hastings.

After the two or three required approximations were generated for the real-time processing application, it was realized that with little additional expense, an entire range of low-order approximations could be generated. This paper presents the results.

For other, more general forms of rational approximations, the reader is referred to Hart, et al. [3].

Received September 9, 1974. $65 \mathrm{D} 15$.

AMS (MOS) subject classifications (1970). Primary 33-04, 33A20, 65D20; Secondary 41 A20,

* The error function $\operatorname{erf}(z)=(2 / \sqrt{\pi}) \int_{0}^{z} e^{-t^{2}} d t$ is related to the normal probability integral by $\operatorname{erf}(z)=1-2 Q(z \sqrt{2})$. 
2. Method. The objective is defined as the following: Find coefficients $\mathrm{c}=$ $\left(c_{1}, \ldots, c_{n}\right)$ such that

$$
\max _{x \in I}\left|\frac{\bar{Q}(x, \mathrm{c})-Q(x)}{w(x)}\right|
$$

is a minimum. For $w(x)=1$, the maximum absolute error is minimized; for $w(x)=$ $Q(x)$, the maximum relative error is minimized.

The problem was approached in the following manner: First, the minimax criterion was loosened to hold over a discrete set $x_{i} \in I, i=1, \ldots, m$. An interval of approximation was then selected along with an appropriate set of $x_{i}$.

The objective can then be stated as: Find $\mathrm{c}=\left(c_{1}, \ldots, c_{n}\right)$ such that $e$ is a minimum where

$$
\left|\frac{\bar{Q}\left(x_{k}, \mathbf{c}\right)-Q\left(x_{k}\right)}{w\left(x_{k}\right)}\right| \leqslant e, \quad k=1, \ldots, m
$$

3. Solution. Suppose we have a nominal solution $\mathbf{c}^{0}$ to the above problem. Then by linearizing $Q\left(x_{k}, \mathrm{c}\right)$ about this nominal solution and noting that $w\left(x_{k}\right)>0$, we obtain

$$
\left|\sum_{i=1}^{n} \delta c_{i} \frac{\partial \bar{Q}\left(x_{k}, \mathrm{c}^{0}\right)}{\partial c_{i}}+\bar{Q}\left(x_{k}, \mathrm{c}^{0}\right)-Q\left(x_{k}\right)\right| \leqslant w\left(x_{k}\right) e
$$

where $\delta c_{i}=c_{i}-c_{i}^{0}$. As suggested by Rabinowitz [4], we rewrite (6) as the following two sets of inequalities:

$$
\begin{gathered}
\sum \delta c_{i} \frac{\partial \bar{Q}}{\partial c_{i}}+\bar{Q}\left(x_{k}, \mathbf{c}^{\mathbf{0}}\right)-Q\left(x_{k}\right) \leqslant w\left(x_{k}\right) e, \quad k=1, \ldots, m, \\
-\left[\sum \delta c_{i} \frac{\partial \bar{Q}}{\partial c_{i}}+\bar{Q}\left(x_{k}, \mathbf{c}^{\mathbf{0}}\right)-Q\left(x_{k}\right)\right] \geqslant-w\left(x_{k}\right) e, \quad k=1, \ldots, m .
\end{gathered}
$$

Equations (7) and (8) can be thought of as a linear programming problem in the $n+1$ variables $\left(\delta c_{1}, \ldots, \delta c_{n}, e\right)$, the objective function being to minimize $e$. When a solution $\delta c$ is found, (6) is re-evaluated using the improved nominal value $c^{(1)}=c^{0}+$ $\delta c$. Since the choice of the nominal value $c^{0}$ was very close to the true value, convergence was always rapid, taking no more than three to five iterations. The iterations were terminated when all changes in coefficients were less than $10^{-15}$.

We now discuss the manner in which $\mathrm{c}^{0}$ was chosen with a simple example. If we choose the approximating function $\bar{Q}$ as follows:

$$
\bar{Q}(x, c, d)=0.5 /(c+d x)^{r},
$$

then Eq. (5) can be written as

$$
-w_{k} e \leqslant 0.5 /\left(c+d x_{k}\right)^{r}-Q\left(x_{k}\right) \leqslant w_{k} e
$$


or

$$
Q\left(x_{k}\right)-w_{k} e \leqslant 0.5 /\left(c+d x_{k}\right)^{r} \leqslant Q\left(x_{k}\right)+w_{k} e .
$$

Note that since $Q\left(x_{k}\right)$ is relatively large and positive and $e$ small, both the left and right hand members of the above inequality will be positive. Raising each member of $(11)$ to the $(-1 / r)$ power, we obtain

$$
\left[Q\left(x_{k}\right)-w_{k} e\right]^{-1 / r} \geqslant(0.5)^{-1 / r}\left(c+d x_{k}\right) \geqslant\left[Q\left(x_{k}\right)+w_{k} e\right]^{-1 / r} .
$$

By using the binomial expansion to first order of the left and right hand members of (12) and multiplying all members by $(0.5)^{1 / r}$, there results

$$
\left(\frac{0.5}{Q\left(x_{k}\right)}\right)^{1 / r}\left(1+\frac{w_{k} e}{r Q\left(x_{k}\right)}\right) \geqslant c+d x_{k} \geqslant\left(\frac{0.5}{Q\left(x_{k}\right)}\right)^{1 / r}\left(1-\frac{w_{k} e}{r Q\left(x_{k}\right)}\right) .
$$

Inequalities (13) are linear in $c, d$, and $e$; and as before for $\delta c$, we can use linear programming to find a solution which minimizes $e$.

4. Results. Approximations were derived for the $n$ in Eq. (3) varying from 3 to 9 and $q$ varying from 0 to 6 . The resulting minimum errors are presented in Figs. 1 through 4. Indicated are the fit criterion used (minimum absolute error and minimum relative error) and the range of validity of the approximation. The value of $n$ can be read off the bottom of the figure, while the $q$ values can be found above the data points. For comparison, methods which require approximately the same amount of computational effort (multiplications) are connected by lines. The optimal approximation for a given amount of computation will then be found at line minimums. This method of display is due to Hastings [2].

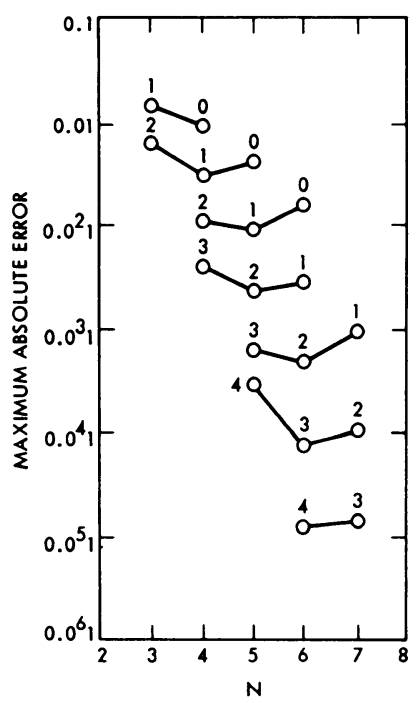

Figure 1. Absolute Error Criterion Range: $0 \leq x<\infty$

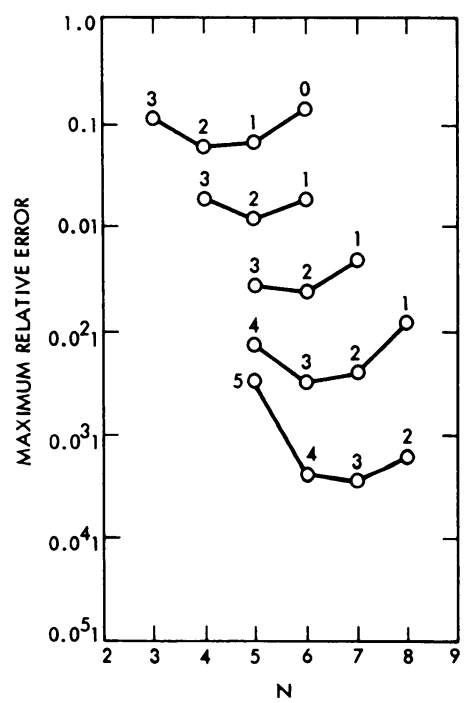

Figure 2. Relative Error Criterion Range: $0 \leq x \leq 3.09$ 


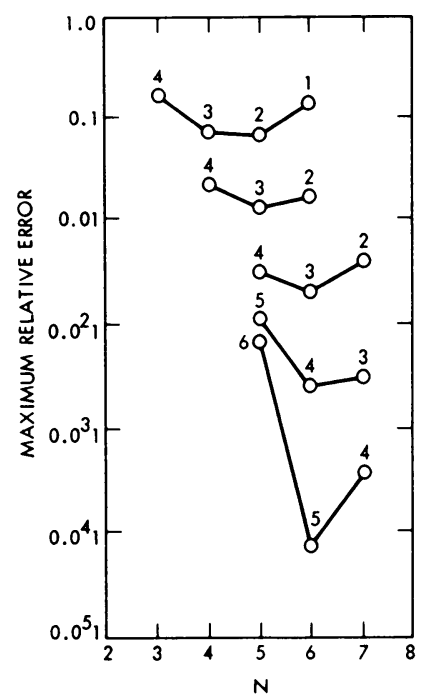

Figure 3. Relative Error Criterion Rąnge: $0 \leq x \leq 4.00$

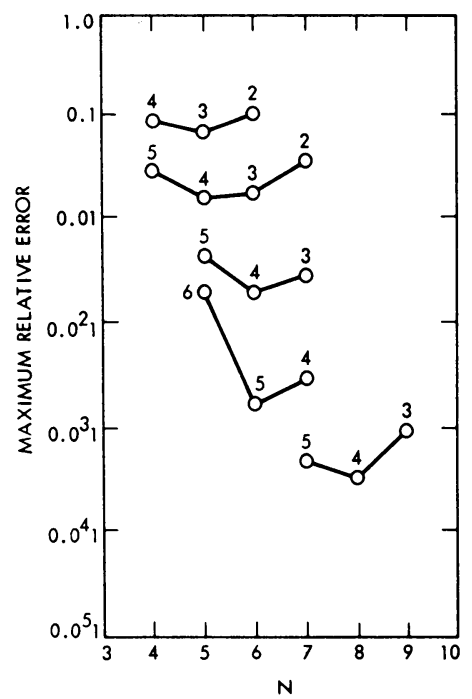

Figure 4. Relative Error Criterion Range: $0 \leq x \leq 5.20$

The approximations which yield the minimum errors described above are presented in Tables 1 through 4. Also given are approximations just to the left of the minimums with errors close to the minimums, as these approximations require one less addition while the slightly reduced accuracy may be acceptable. Further approximations were also derived with one coefficient constrained to zero. This reduces the computational cost by one addition while increasing the error somewhat. Though these approximations were not plotted in Figs. 1 to 4, when they compared favorably

TABLE 1. Coefficients for Approximations Valid over $0 \leq \mathrm{x}<\infty$. (Absolute Error Minimized)

\begin{tabular}{|c|c|c|c|c|c|c|c|}
\hline \multirow{2}{*}{$\begin{array}{c}N=4 \quad Q=0 \\
\text { ABS ERR }=8.89 \mathrm{E}-3 \\
0.98326277 \mathrm{E}-0 \\
0.13447918 \mathrm{E}+1 \\
-0.15765831 \mathrm{E}+1 \\
0.25263428 \mathrm{E}+1\end{array}$} & \multicolumn{2}{|c|}{$\begin{array}{c}N=4 \quad Q=0 \\
\text { ABS ERR }=1.13 \mathrm{E}-2\end{array}$} & \multicolumn{2}{|c|}{$\begin{array}{c}N=4 \quad Q=1 \\
\text { ABS ERR }=3.04 E-3\end{array}$} & \multicolumn{2}{|c|}{$\begin{array}{c}\mathrm{N}=4 \quad Q=1 \\
\mathrm{ABS} \mathrm{ERR}=3.22 \mathrm{E}-3\end{array}$} & \multirow{2}{*}{$\begin{array}{c}N=4 \quad Q=2 \\
A B S E R=1.07 E-3 \\
0.99947268 \mathrm{E}-0 \\
0.21268088 \mathrm{E}-0 \\
0.55642184 \mathrm{E}-1 \\
0.54262629 \mathrm{E}-1\end{array}$} \\
\hline & \multicolumn{2}{|c|}{$\begin{array}{l}1.02276817 \mathrm{E}-0 \\
0.68874886 \mathrm{E}-0 \\
0.0 \\
0.16777473 \mathrm{E}+1\end{array}$} & \multicolumn{2}{|c|}{$\begin{array}{r}0.99702758 \mathrm{E}-0 \\
0.48032748 \mathrm{E}-0 \\
-0.50749808 \mathrm{E}-1 \\
0.35180598 \mathrm{E}-0\end{array}$} & \multicolumn{2}{|c|}{$\begin{array}{l}1.00055503 \mathrm{E}-0 \\
0.45095789 \mathrm{E}-0 \\
0.0 \\
0.32985306 \mathrm{E}-0\end{array}$} & \\
\hline \multicolumn{2}{|c|}{$\begin{array}{c}N=5 \quad Q=1 \\
\text { ABS } \mathrm{ERR}=8 . ? 9 \mathrm{E}-4\end{array}$} & $\begin{array}{c}\mathrm{N}=5 \\
\mathrm{ABS} \text { ERR }=2\end{array}$ & $\begin{array}{c}Q=2 \\
2.23 E-4\end{array}$ & \multicolumn{2}{|c|}{$\begin{array}{c}N=5 \quad Q=2 \\
\text { ABS ERR }=2.31 \quad E-4\end{array}$} & \multicolumn{2}{|c|}{$\begin{array}{c}N=5 \quad Q=3 \\
\text { ABS ERR }=6.52 \mathrm{E}-5\end{array}$} \\
\hline \multicolumn{2}{|c|}{$\begin{array}{r}1.00086821 \mathrm{E}-0 \\
0.36630736 \mathrm{E}-0 \\
0.41183061 \mathrm{E}-0 \\
-0.18627441 \mathrm{E}-0 \\
0.17767962 \mathrm{E}-0\end{array}$} & \multicolumn{2}{|c|}{$\begin{array}{r}1.00010947 \mathrm{E}-0 \\
0.19566657 \mathrm{E}-0 \\
0.11816079 \mathrm{E}-0 \\
-0.21320100 \mathrm{E}-2 \\
0.20162569 \mathrm{E}-1\end{array}$} & \multicolumn{2}{|c|}{$\begin{array}{l}1.00001244 \mathrm{E}-0 \\
0.19670088 \mathrm{E}-0 \\
0.11559155 \mathrm{E}-0 \\
0.0 \\
0.19617429 \mathrm{E}-1\end{array}$} & \multicolumn{2}{|c|}{$\begin{array}{l}1.00001431 \mathrm{E}-0 \\
0.99230111 \mathrm{E}-1 \\
0.47182012 \mathrm{E}-1 \\
0.50442815 \mathrm{E}-2 \\
0.27727664 \mathrm{E}-2\end{array}$} \\
\hline \multicolumn{2}{|c|}{$\begin{array}{c}N=6 \quad Q=2 \\
\text { ABS ERR }=4.61 \quad E-5\end{array}$} & \multicolumn{2}{|c|}{$\begin{array}{c}N=6 \quad Q=3 \\
\text { ABS ERR }=8.02 \text { E-6 }\end{array}$} & \multicolumn{2}{|c|}{$\begin{array}{cc}N=6 & Q=3 \\
\text { ABS ERR } & =9.50 \mathrm{E}-6\end{array}$} & \multicolumn{2}{|c|}{$\begin{array}{cc}N=6 & Q=4 \\
\text { ABS ERR } & =1.20 \mathrm{E}-6\end{array}$} \\
\hline \multicolumn{2}{|c|}{$\begin{array}{r}0.9999772773 \mathrm{E}-0 \\
0.2004847193 \mathrm{E}-0 \\
0.9277989663 \mathrm{E}-1 \\
0.4102510451 \mathrm{E}-1 \\
-0.7732107617 \mathrm{E}-2 \\
0.5919110319 \mathrm{E}-2\end{array}$} & \multicolumn{2}{|c|}{$\begin{array}{r}0.9999980543 \mathrm{E}-0 \\
0.9981881352 \mathrm{E}-1 \\
0.4423299153 \mathrm{E}-1 \\
0.9807669823 \mathrm{E}-2 \\
-0.1535053920 \mathrm{E}-3 \\
0.5910199800 \mathrm{E}-3\end{array}$} & \multicolumn{2}{|c|}{$\begin{array}{l}1.0000020249 \mathrm{E}-0 \\
0.9975397369 \mathrm{E}-1 \\
0.4446283166 \mathrm{E}-1 \\
0.9511996161 \mathrm{E}-2 \\
0.0 \\
0.5638925614 \mathrm{E}-3\end{array}$} & \multicolumn{2}{|c|}{$\begin{array}{r}0.9999998582 \mathrm{E}-0 \\
0.4987385796 \mathrm{E}-1 \\
0.2109811045 \mathrm{E}-1 \\
0.3372948927 \mathrm{E}-2 \\
-0.5172897742 \mathrm{E}-4 \\
0.8569579420 \mathrm{E}-4\end{array}$} \\
\hline
\end{tabular}


TABLE 2. Coefficients for Approximations Valid over $0 \leq \mathrm{x} \leq 3.09$ (Relative Error Minimized)

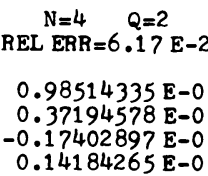

0.9994503392 E-O $0.2132836195 \mathrm{E}-0$ $0.4538762171 \mathrm{E}-1$ $0.1001012612 \mathrm{E}-0$ $-0.3678315622 \mathrm{E}-1$ $0.1076796149 \mathrm{E}-1$
$\mathrm{N}=4 \quad \mathrm{Q}=3$
REL ERR $=1.92 \mathrm{E}-2$

0.99766167 E-0

$0.12422288 \mathrm{E}-0$

$0.77418010 \mathrm{E}-2$

$0.24195179 \mathrm{E}-1$
$\begin{array}{cc}N=5 & Q=2 \\ \text { REL ERR }=1 & .25 \mathrm{E}-2\end{array}$

$1.00310973 \mathrm{E}-0$

$0.14518284 \mathrm{E}-0$

0.24306692 E-0

-0.96327447 E-1

$0.1+1493605 \mathrm{E}-1$

$N=6 \quad Q=4$

REL ERR $=3.86 \mathrm{E}-5$

$0.9999975888 \mathrm{E}-0$ $0.4992364716 \mathrm{E}-1$

$0.2092978887 \mathrm{E}-1$

$0.3566148574 \mathrm{E}-2$

$0.3566148574 \mathrm{E}-2$
$-0.1397344692 \mathrm{E}-3$

$0.9934962303 \mathrm{E}-4$
$N=5 \quad Q=3$

REL ERR $=2.55 \mathrm{E}-3$

1.00033090 E-0

$0.94263331 \mathrm{E}-1$

$0.58802340 \mathrm{E}-1$

$-0.33152891 \mathrm{E}-2$

$0.45893284 \mathrm{E}-2$
0.9999629152 E-0 $0.1006157723 \mathrm{E}-0$ $0.1309929761 \mathrm{E}-1$ $-0.1694672049 \mathrm{E}-2$ $0.8365931999 \mathrm{E}-3$

\begin{abstract}
$\mathrm{N}=7 \quad \mathrm{Q}=3$ REL ERR $=3.63 \mathrm{E}-5$

1.0000043564 E-0 0.9959491 608 E-1 0.4548994791 E-1 0.7297927613 E-2 $0.2044973272 \mathrm{E}-2$ $-0.2688525816 \mathrm{E}-$

$0.1220418799 \mathrm{E}-3$
\end{abstract}

TABLE 3. Coefficients for Approximations Valid over $0 \leq x \leq 4.00$ (Relative Error Minimized)

\begin{tabular}{|c|c|c|c|}
\hline $\begin{array}{c}N=4 \quad Q=3 \\
\text { REL ERR }=7.03 E-2\end{array}$ & $\begin{array}{c}N=4 \quad Q=3 \\
\text { REL ERR }=1.02 \mathrm{E}-1\end{array}$ & $\begin{array}{c}N=5 \quad Q=2 \\
\text { REL } \mathrm{ERR}=6.42 \mathrm{E}-2\end{array}$ & $\begin{array}{c}N=5 \quad Q=3 \\
\text { REL ERR }=1.29 \mathrm{E}-2\end{array}$ \\
\hline $\begin{array}{r}0.99159673 \mathrm{E}-0 \\
0.17157632 \mathrm{E}-0 \\
-0.40278659 \mathrm{E}-1 \\
0.35725676 \mathrm{E}-1\end{array}$ & $\begin{array}{l}1.01343935 \mathrm{E}-0 \\
0.10575969 \mathrm{E}-0 \\
0.0 \\
0.29235914 \mathrm{E}-1\end{array}$ & $\begin{array}{r}1.01656534 \mathrm{E}-0 \\
-0.29167615 \mathrm{E}-1 \\
0.58796837 \mathrm{E}-0 \\
-0.30295449 \mathrm{E}-0 \\
0.78591661 \mathrm{E}-1\end{array}$ & $\begin{array}{r}1.0 \\
0.7 \\
0.8 \\
-0.1 \\
0.7\end{array}$ \\
\hline $\begin{array}{l}N=5 \quad Q=4 \\
\mathrm{FRR}=3.18 \mathrm{E}-3\end{array}$ & 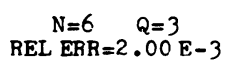 & $\begin{array}{c}N=6 \quad Q=4 \\
\text { REL } \mathrm{ERR}=2.61 \mathrm{E}-4\end{array}$ & $\begin{array}{c}N=6 \quad Q=5 \\
\text { REL } \text { ERR }=7.04 \text { E-6 }\end{array}$ \\
\hline $\begin{array}{l}1.00016069 \mathrm{E}-0 \\
0.47512354 \mathrm{E}-1 \\
0.25955649 \mathrm{E}-1 \\
0.0 \\
0.87461784 \mathrm{E}-3\end{array}$ & $\begin{array}{r}0.99975192 \mathrm{E}-0 \\
0.10438503 \mathrm{E}-0 \\
0.31073947 \mathrm{E}-1 \\
0.22824383 \mathrm{E}-1 \\
-0.53407894 \mathrm{E}-2 \\
0.13078095 \mathrm{E}-2\end{array}$ & $\begin{array}{r}0.99998392 \mathrm{E}-0 \\
0.50155550 \mathrm{E}-1 \\
0.20321174 \mathrm{E}-1 \\
0.41149238 \mathrm{E}-2 \\
-0.33900954 \mathrm{E}-3 \\
0.12443051 \mathrm{E}-3\end{array}$ & $\begin{array}{r}0.9999997873 \mathrm{E}-0 \\
0.2493389125 \mathrm{E}-1 \\
0.1026041229 \mathrm{E}-1 \\
0.1386662649 \mathrm{E}-2 \\
-0.7799896470 \mathrm{E}-4 \\
0.1953637532 \mathrm{E}-4\end{array}$ \\
\hline
\end{tabular}

TABLE 4. Coefficients for Approximations Valid over $0 \leq \mathrm{x} \leq 5.20 \quad$ (Relative Error Minimized)

$$
\begin{aligned}
& \begin{array}{l}
N=4 \quad Q=4 \\
\text { ER }
\end{array} \\
& \text { REL ERR }=8 \cdot 50 \mathrm{E}- \\
& 0.99498362 \text { E-0 } \\
& 0.81690469 \mathrm{E}-1 \\
& -0.69190469 \mathrm{E}-2 \\
& 0.97932565 \mathrm{E}-2 \\
& \mathrm{~N}=5 \quad \mathrm{Q}=3 \\
& \text { REL ERR }=6.79 \mathrm{E}-2 \\
& 1.00867395 \mathrm{E}-0 \\
& 0.11885495 \mathrm{E}-1 \\
& 0.18389763 \mathrm{E}-0 \\
& -0.61961072 \mathrm{E}-1 \\
& 0.12983665 \mathrm{E}-1 \\
& N=6 \quad Q=4 \\
& \text { REL FRR }=1.90 \mathrm{E}-3 \\
& N=6 \quad Q=5
\end{aligned}
$$

$$
\begin{gathered}
N=5 \quad Q=4 \\
\text { REL ERR }=1.52 \mathrm{E}-2 \\
1.00089169 \mathrm{E}-0 \\
0.41079083 \mathrm{E}-1 \\
0.34686804 \mathrm{E}-1 \\
-0.36787667 \mathrm{E}-2 \\
0.13477384 \mathrm{E}-2
\end{gathered}
$$

$\mathrm{N}=8 \quad \mathrm{Q}=4$

REL ERR $=3.37 \mathrm{E}-5$

0.9999980589 E-0

$0.4992025890 \mathrm{E}-1$

$0.2091639596 \mathrm{E}-1$

$0.3638516531 \mathrm{E}-2$

$-0.2437065631 \mathrm{E}-3$

$0.1648858949 \mathrm{E}-3$

$-0.1003371134 \mathrm{E}-4$

$0.2078875419 \mathrm{E}-5$ 
with one of the plotted approximations they were included in the tables.

For most rapid evaluation, the approximations should not be used directly as they stand in the tables, but rather, the coefficient of the highest power of $x$ should be normalized to unity. Thus, for example, the approximation

$$
Q(x) \approx \frac{0.5}{\left(1.000555+0.450958 x+0.329853 x^{3}\right)^{2}}
$$

should be evaluated as

$$
Q(x) \approx \frac{4.5954587}{\left(3.033335+1.367148 x+x^{3}\right)^{2}}
$$

which requires one less multiplication.

Further savings can be obtained by economizing polynomials of degree greater than 3. For example, the quartic $P(x)=x^{4}+A x^{3}+B x^{2}+C x+D$ can be evaluated by

$$
\begin{aligned}
P_{1} & =x(x+a) \\
P(x) & =\left(P_{1}+x+b\right)\left(P_{1}+c\right)+d
\end{aligned}
$$

where

$$
\begin{aligned}
& a=(A-1) / 2, \\
& c=C-a[B-a(a+1)], \\
& b=B-a(a+1)-c, \\
& d=D-b c .
\end{aligned}
$$

Evaluation of the quartic by (16) requires one less multiplication than the usual nested multiplication algorithm at a cost of one extra addition.

The quintic can be evaluated from the quartic solution by the factorization

$$
\begin{aligned}
P(x) & =x^{5}+A x^{4}+B x^{3}+C x^{2}+D x+E \\
& =x\left(x^{4}+A x^{3}+B x^{2}+C x+D\right)+E .
\end{aligned}
$$

Similarly, the sixth-order polynomial

$$
P(x)=x^{6}+A x^{5}+B x^{4}+C x^{3}+D x^{2}+E x+F,
$$

can be evaluated by the following algorithm:

$$
\begin{aligned}
P_{1} & =x(x+a) \\
P_{2} & =\left(P_{1}+x+b\right)\left(P_{1}+c\right), \\
P(x) & =\left(P_{2}+d\right)\left(P_{1}+e\right)+f
\end{aligned}
$$

which requires two less multiplications than nested multiplication at the cost of a single extra addition.

Caution is recommended on the use of polynomial economization. Though not usually a problem for the approximations derived here, it is sometimes necessary to 
carry greater precision in the calculation and use of the modified coefficients $(a, b, c$, etc.) than is immediately apparent. For this reason, all coefficients in the tables are given to greater precision than needed for straightforward nested multiplication evaluation.

The reader is referred to [5], [6], [7], [8] for further information on polynomial economization.

5. Conclusion. A number of cost-effective low-order approximations have been derived for the normal probability integral by linear programming minimax techniques. These approximations are all of the reciprocal polynomial type. Methods have been presented for the efficient evaluation of these polynomials.

Jet Propulsion Laboratory

California Institute of Technology

4800 Oak Grove Drive

Pasadena, California 91103

1. D. G. CARTA, "Low-order approximations for real time simulation," Record of Proc., The Seventh Annual Simulation Symposium, Tampa, Florida, March, 1974, pp. 241-252.

2. C. HASTINGS, Approximations for Digital Computers, Princeton Univ. Press, Princeton, N. J., 1955, pp. 93, 185. MR 16, 963.

3. J. F. HART, et al., Computer Approximations, Wiley, New York, 1968.

4. P. RABINOWITZ, "Applications of linear programming to numerical analysis," SIAM Rev., v. 10, 1968, pp. 121-159. MR 37 \#2397.

5. J. TODD (Editor), Survey of Numerical Analysis, McGraw-Hill, New York, 1962, pp. 3-4. MR 24 \#B1271.

6. E. G. BELAGA, "Some problems involved in the calculation of polynomials," Dokl. Akad. Nauk SSSR, v. 123, 1958, pp. 775-777. (Russian) MR 21 \#3935.

7. L. A. LJUSTERNIK, et al., Mathematical Analysis. Computation of the Elementary Functions, Fizmatgiz, Moscow, 1963; English transl., Handbook for Computing Elementary Functions, Pergamon Press, Oxford and New York, 1965. MR 28 \#1733; 32 \#584.

8. D. E. KNUTH, The Art of Computer Programming. Vol. 2: Seminumerical Algorithms, Addison-Wesley, Reading, Mass., 1969. MR 44 \#3531. 